\title{
Longitudinal Relationship Between Metabolic Syndrome and Periodontal Disease Among Japanese Adults Aged $\geq 70$ Years: The Niigata Study
}

Masanori Iwasaki, ${ }^{* \dagger}$ Misuzu Sato ${ }^{\dagger}$ Kumiko Minagawa, ${ }^{\dagger}$ Michael C. Manz, ${ }^{\ddagger}$ Akihiro Yoshihara,, and Hideo Miyazaki ${ }^{\dagger}$

Background: There has been little evaluation in longitudinal epidemiologic studies of the effect of metabolic syndrome (MetS) on periodontal status. The specific aim of this longitudinal study is to investigate whether MetS in the Japanese population could be a risk factor for periodontal disease.

Methods: A total of 125 older adults from Japan for whom data were available for the years 2003 to 2006 were selected for the current study. Full-mouth periodontal status, measured as clinical attachment level (CAL), was recorded at baseline and in follow-up examinations. Development of periodontal disease was considered to be $\geq 2$ teeth demonstrating a longitudinal loss of proximal attachment of $\geq 3 \mathrm{~mm}$ at the follow-up dental examination. A multivariable Poisson regression model with robust error variance was used to evaluate the association of MetS defined by the modified National Cholesterol Education Program Adult Treatment Panel III criteria with development of periodontal disease. Adjustments for sex, income, education, smoking status, number of teeth at baseline, mean CAL at baseline, pattern of visits to a dentist, and brushing frequency were considered.

Results: The prevalence of MetS was 21.6\% (27/125). Study participants with MetS were approximately 2.6 times more likely to develop periodontal disease (adjusted relative risk $2.58,95 \%$ confidence interval 1.17 to 5.67 ) after simultaneous adjustment for other covariates.

Conclusions: These findings support the hypothesis that MetS may be a risk factor for periodontal disease in older Japanese individuals. Additional studies with larger, more diverse populations and more complete information are needed to substantiate the findings. J Periodontol 2015;86:491-498.

\section{KEY WORDS}

Aged; epidemiology; longitudinal studies; metabolic syndrome $\mathrm{X}$; oral medicine; periodontal diseases.

\footnotetext{
* Division of Community Oral Health Development, Kyushu Dental University, Kitakyushu, Japan.

† Division of Preventive Dentistry, Department of Oral Health Science, Niigata University Graduate School of Medical and Dental Sciences, Niigata, Japan.

† Department of Cariology, Restorative Sciences, and Endodontics; School of Dentistry; University of Michigan; Ann Arbor, MI.

$\S$ Division of Oral Science for Health Promotion, Department of Oral Health and Welfare, Niigata University Graduate School of Medical and Dental Sciences.
}

$M$ etabolic syndrome (MetS) is characterized as a clustering of metabolic disorders: abdominal obesity, elevated blood pressure (BP), dyslipidemia, and hyperglycemia. ${ }^{1}$ MetS contributes to chronic inflammation and to an increased risk of systemic diseases such as cardiovascular disease and type 2 diabetes. ${ }^{2}$ MetS is becoming increasingly prevalent among many different populations worldwide, including Japanese. The estimated number of people aged $\geq 40$ years with MetS is 10.7 million (18.4\%) in Japan. ${ }^{3}$

Periodontal disease affects $>40 \%$ of adults aged $\geq 45$ years in Japan ${ }^{4}$ and is characterized by chronic inflammation of tooth-supporting tissues. It has been suggested that MetS and periodontal disease may share a pathogenic background through oxidative stress/mitochondrial dysfunction mechanisms. ${ }^{5,6}$ Individuals with MetS may have greater susceptibility to periodontal disease. In fact, previous epidemiologic studies have demonstrated that patients with MetS had a higher risk of poor periodontal status. ${ }^{7-15}$ However, most of the studies $^{7-13,15}$ conducted to date had a crosssectional design, which prevents the establishment of an unambiguous causeeffect relationship.

Further investigation of the relationship between MetS and periodontal disease 
in a longitudinal study is important to gain insight into MetS as a potential risk factor for periodontal disease. The hypothesis of the present study is that MetS was associated with an increased risk of periodontal disease. This study aims to determine if there were longitudinal associations of MetS with periodontal disease in community-dwelling older Japanese individuals.

\section{MATERIALS AND METHODS}

\section{Study Design, Setting, and Participants}

The current investigation is a subset study of the Niigata Study ${ }^{16}$ over the period 2003 to 2006 . Briefly, the Niigata Study is a prospective community-based study initiated in 1998 to evaluate relationships between the systemic health status and history of dental diseases. In April 1998, all 4,542 Niigata citizens aged 70 years (2,099 men and 2,443 women) were sent a written request to participate in the survey. The invitation was mailed again to non-respondents 3 weeks later; $81.4 \%(3,695 / 4,542)$ responded positively to participate in the survey. Considering the availability of resources, examination appointments could be arranged for 600 individuals. The final study sample was randomly recruited from several areas of Niigata to have an approximately equal number of men $(n=306)$ and women $(n=294)$ (Fig. 1).

At the baseline of the current study (2003), dental and medical examinations, an interview, and an anthropometric evaluation were conducted, and 163 individuals were selected according to the following criteria. Inclusion criteria: 1 ) original members of the Niigata study; 2) individuals who had at least 10 teeth; ${ }^{15,17}$ and 3) individuals without periodontal disease (presence of proximal attachment loss $\geq 5$ $\mathrm{mm}$ in $\geq 30 \%$ of teeth present was defined as positive ${ }^{18}$ ). Exclusion criteria: 1) individuals who were hospitalized; 2) individuals who were living in nursing homes; and 3) individuals who had missing data. Three years later, study entrants were invited to the follow-up examinations, including dental assessment, in which 38 were not available to participate. Data were therefore analyzed for the 125 participants completing the follow-up examination in 2006.

This study was conducted according to the guidelines laid down in the Declaration of Helsinki, and all procedures involving human individuals were approved by the Ethics Committee of the Faculty of Dentistry, Niigata University. Written informed consent was obtained from all study participants.

\section{Dental Examination}

The dental examination was conducted at baseline and follow-up under sufficient illumination using artificial light by four trained dentists (Hiroshi Ogawa, Takayuki Yamaga, Toshinobu Hirotomi, and Naoko
Takano, all of Niigata University Graduate School of Medical and Dental Sciences, Niigata, Japan) who assessed clinical attachment level (CAL) and number of teeth (including third molars). ${ }^{19}$ For CAL, a pressuresensitive probell was used to measure to the nearest millimeter the distance from the cemento-enamel junction (CEJ) to the base of the pocket, at six sites of all remaining natural teeth. At sites where the CEJ was unclear because it was concealed by the margin of a crown restoration, the position of the CEJ was estimated using adjacent landmarks and dental anatomy. When the CEJ could not be estimated, the site was excluded from the examination. Before the examination, calibrations were done at the Niigata University Medical \& Dental Hospital. Intra- and interexaminer reliability of CAL within $1 \mathrm{~mm}$ was confirmed using both percent agreement $(86.6 \%$ to 95.9\%) and $\kappa$ statistic (0.79 to 0.93).

\section{Medical Examination, Anthropometric Evaluation, and Interview}

Fasting blood samples were drawn for the measurement of triglycerides, high-density lipoprotein cholesterol (HDL-C), and hemoglobin A1c (HbA1c). $\mathrm{BP}$ recordings were obtained from the right arm of the participants in a sitting position after 5 minutes of rest. Anthropometric evaluation included measurements of height and weight to calculate body mass index (BMI). A standardized questionnaire was completed, covering treatment for hypertension or diabetes, smoking status (with those reporting any smoking history classified as smokers), socio-economic status (household income and years of school attendance), and oral health-related behavior (brushing frequency [ $<2$ or $\geq 2$ times/day] and pattern of visits to a dentist [regularly or episodically]). Lower income was defined as annual household income $<2,000,000$ Japanese yen (US \$16,667; US \$1 = 120 Japanese yen; average annual household income = $5,890,000$ Japanese yen in 2003). Lower education was defined as school attendance $\leq 8$ years.

\section{Description of Outcome Variables}

The primary outcome for the analyses included the development of periodontal disease. Development of periodontal disease was defined by using criteria proposed in the European Workshop in Periodontology. ${ }^{18}$ Study participants who had $\geq 2$ teeth demonstrating a longitudinal loss of proximal attachment $\geq 3 \mathrm{~mm}$ at the follow-up dental examination were considered to have developed periodontal disease.

\section{Description of Principal Exposure Variable}

The principal exposure variable was MetS. MetS was defined by using criteria recommended in the

\| Vivacare TPS probe, Vivadent, Schaan, Liechtenstein. 


\section{Niigata study initiated}

All 4,542 Niigata citizens aged 70 years old were sent a written request to participate in the survey.

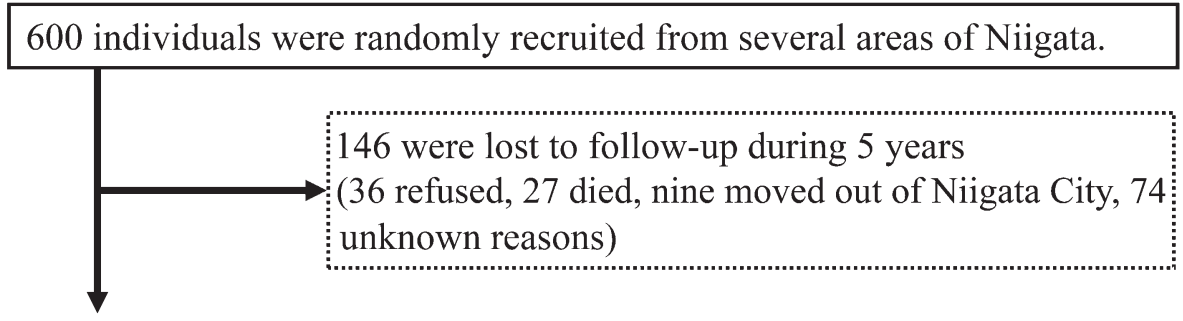

2003 baseline survey for the current study

Eligible participants

= original members of Niigata study: 212

(118 having $<10$ teeth and 124 having periodontal disease were excluded)

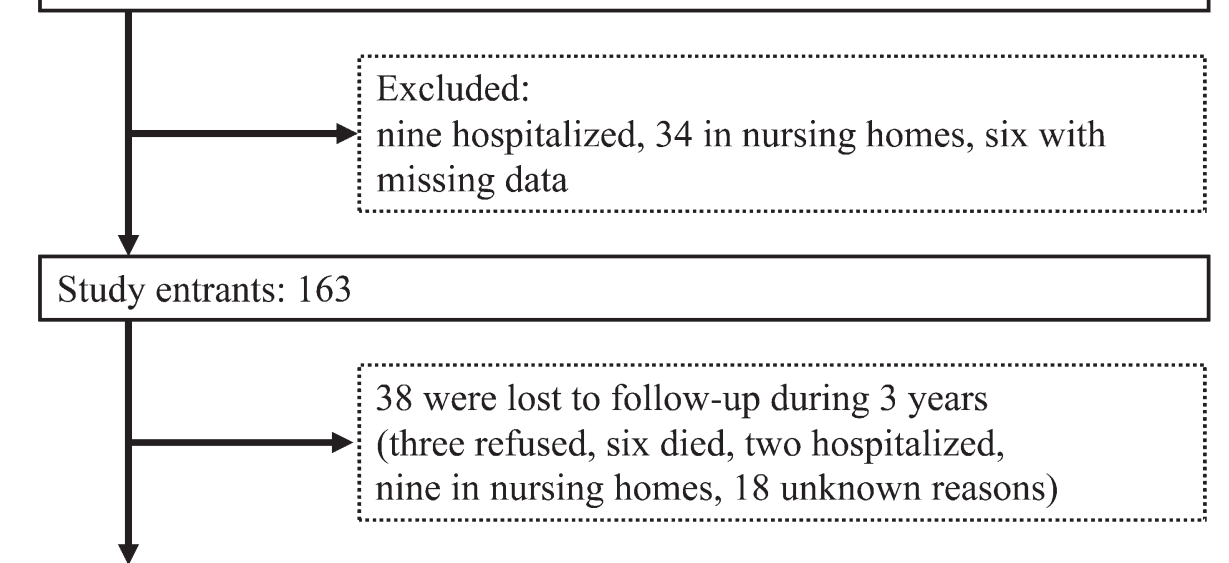

2006 follow-up survey for the current study

Study participants: 125

Participants/entrants $=76.7 \%$

\section{Figure I.}

Flow diagram of the study.

National Cholesterol Education Program Adult Treatment Panel III guidelines, with modification. ${ }^{1,20}$ Abdominal obesity was defined as BMI $\geq 25 \mathrm{~kg} / \mathrm{m}^{2}$. $\mathrm{BMI}$ was used as a surrogate for waist circumference to define abdominal obesity. ${ }^{11,15}$ Elevated BP was defined as systolic/diastolic BP of $\geq 130 / 85 \mathrm{~mm} \mathrm{Hg}$ and/or the current use of antihypertensive medications. Hypertriglyceridemia was defined as serum triglycerides $\geq 150 \mathrm{mg} / \mathrm{dL}$. Low HDL-C was defined as serum HDL-C levels $<40 \mathrm{mg} / \mathrm{dL}$ in men and $<50 \mathrm{mg} /$ $\mathrm{dL}$ in women. Hyperglycemia was defined as HbA1c $\geq 6.0 \%$ and/or the current use of antidiabetic medi- cations. HbA1c was used as a surrogate for fasting plasma glucose to define hyperglycemia. ${ }^{21}$ MetS was defined as the presence of three or more of these components. ${ }^{1}$

\section{Statistical Analyses}

Before the principal analysis to address the main question of interest, differences in socioeconomic status, health behavior, and health status were assessed among participants and the individuals who were lost to follow-up.

The participants' variables were described using the frequency distribution for categorical variables and the mean $\pm S D$ for continuous variables. The $\chi^{2}$ test for categorical variables and Student $t$ test for continuous variables were used to test differences in the percentages and the means of baseline characteristics between individuals with and without MetS.

Tests of the study's principal hypothesis that MetS is associated with an increased risk of developing periodontal disease included univariable and multivariable Poisson regression modeling with robust error variance. Multivariable models were adjusted for potential confounders: sex, income, education, smoking status, number of the teeth at baseline, mean CAL at baseline, pattern of visits to a dentist, and brushing frequency. The authors also tested interaction terms for each of the third variables with MetS. Interaction terms found not to be statistically significant were not included in the model.

Additionally, participants were divided into three groups according to the number of MetS components; zero to one component, two components, and $\geq 3$ components. Tests for trend were performed by fitting the three-category MetS variable in its continuous form in the regression models.

An $\alpha=0.05$ level was considered statistically significant for all analyses. All calculations and statistical analyses were performed using statistical software. "I

Il STATA v.13, Stata Corp., College Station, TX. 


\section{RESULTS}

There were no significant differences in selected characteristics between study participants and those lost to follow-up (data not shown).

Table 1 lists baseline characteristics of the study population. The prevalence of MetS was 21.6\% (27/ 125). Participants with MetS had significantly higher mean values of $\mathrm{BMI}$, BP, serum triglycerides, and $\mathrm{HbA} 1 \mathrm{c}$ and lower mean values of serum HDL-C than those without MetS. The frequency of antidiabetic medication use was higher in individuals with MetS than in those without MetS.

Table 2 shows the results from the Poisson regression analyses of developing periodontal disease. MetS was associated significantly with an increased risk of developing periodontal disease. The crude relative risk (RR) of developing periodontal disease for participants with MetS was 2.23 (95\% confidence interval [CI], 1.03 to 4.84) compared with those without MetS. MetS remained significantly associated with a higher risk of developing periodontal disease after simultaneously adjusting for other covariates. In the multivariable model, the adjusted RR for participants with MetS was 2.58 (95\% CI, 1.17 to 5.67). In addition, there was a trend toward higher risk of developing periodontal disease with a greater number of MetS components ( $P$ for trend in the multivariable model $=0.04$ ) .

When testing the independent associations of each individual MetS component with the development of periodontal disease (Table 2), no significant and consistent associations were found among each MetS component and the development of periodontal disease. In the crude model, participants with abdominal obesity were significantly associated with a higher risk of developing periodontal disease compared with those without abdominal obesity (crude RR, 2.18; 95\% CI, 1.01 to 4.70 ); however, this association disappeared after multivariable adjustment.

\section{DISCUSSION}

The authors observed a significant association between MetS and development of periodontal disease after controlling for other important baseline health characteristics in multivariable Poisson regression analysis. Individuals with MetS were more likely to develop periodontal disease than individuals without MetS. By including a homogeneous group of older Japanese restricted to the same age at baseline in the survey, the influences of variations in age and race on the results were eliminated. The findings of the present study concur with recent cross-sectional studies that demonstrated a significant association between MetS and poor periodontal health. 7,9-13,15

When the components of MetS were examined separately, there were no statistically significant associations of each MetS component with the risk of developing periodontal disease. This was partially explained by the fact that each individual component includes a variety of conditions, ranging from mild to severe, resulting in underestimation of the association between MetS components and periodontal disease. A combination of multiple risk factors (abdominal obesity, elevated BP, dyslipidemia, and hyperglycemia) can lead to the development of periodontal disease.

Evidence suggests that MetS and periodontal disease may share many causative factors. ${ }^{5,22}$ MetS is a proinflammatory state derived from excessive caloric intake, overnutrition, and other chronic inflammatory conditions. ${ }^{23}$ Abnormal proinflammatory cytokines and adipocytokine production have been observed in patients with MetS. ${ }^{5,24,25}$ It is generally accepted that an inflammatory state exists and is associated with periodontal disease as well, ${ }^{26}$ and proinflammatory cytokines can lead to progressive breakdown of the periodontal tissue. ${ }^{27}$ Adipokines are important in glucose homeostasis, which is known to play a role in obesity and insulin resistance. Both of these have been postulated to be primary contributors to the pathogenesis of MetS. Obesity and insulin resistance are significant risk factors for periodontal disease as well. ${ }^{28,29}$ These findings suggest that individuals with MetS may tend to be more susceptible to periodontal disease in the presence of periodontal pathogens.

Furthermore, it is increasingly recognized that oxidative stress plays an important role in inflammation-based chronic diseases. ${ }^{22}$ Oxidative stress is a consequence of persistent imbalance between the production of reactive oxygen species (ROS) and antioxidant capacity. The main source of cellular ROS is mitochondria. Mitochondrial ROS production is a tightly controlled process that plays a role in the maintenance of cellular oxidative homeostasis and propagation of cellular signaling pathways. ${ }^{30}$ This mechanism is affected by external stimulation with inflammation. The proinflammatory state can lead to an increase in oxidative stress. Altered ROS production, a compromised antioxidant system, and mitochondrial dysfunction are observed in MetS. ${ }^{31}$ Oxidative stress has been demonstrated to alter the structure or function of cells and organisms, ${ }^{32}$ which could contribute to periodontal tissue breakdown as well. ${ }^{22,32,33}$ Elevated oxidative stress marker levels, lower antioxidant capacity, ${ }^{34}$ and increased levels of DNA damage markers ${ }^{35}$ were observed in periodontal disease. Recently, lipopolysaccharides of Porphyromonas gingivalis were reported to be associated with high mitochondrial ROS, low coenzyme Q10 levels, and mitochondrial dysfunction. ${ }^{36}$ Therefore, lipopolysaccharide-mediated mitochondrial dysfunction could 
Table I.

\section{Baseline Characteristics of the Study Participants With and Without MetS [mean $\pm \mathrm{SD}$ or $\mathrm{n}(\%)]$}

\begin{tabular}{|c|c|c|c|}
\hline \multirow[b]{2}{*}{ Variable } & \multicolumn{2}{|c|}{ MetS } & \multirow[b]{2}{*}{$p^{*}$} \\
\hline & No $(n=98)$ & Yes $(n=27)$ & \\
\hline No. of teeth & $23.8 \pm 5.1$ & $22.0 \pm 5.3$ & 0.10 \\
\hline Mean CAL (mm) & $2.7 \pm 0.6$ & $2.9 \pm 0.8$ & 0.12 \\
\hline \multicolumn{4}{|l|}{ Sex } \\
\hline Men & $45(45.9)$ & $10(37.0)$ & 0.41 \\
\hline Women & $53(54.1)$ & $17(63.0)$ & \\
\hline Lower income & $13(13.3)$ & $5(\mid 8.5)$ & 0.49 \\
\hline Lower education & $36(36.7)$ & $13(48.2)$ & 0.28 \\
\hline Current use of antihypertensive medications & $23(23.5)$ & II (40.7) & 0.07 \\
\hline Triglycerides (mg/dL) & $1 \mid 4.0 \pm 57.8$ & $203.1 \pm 70.5$ & $<0.001$ \\
\hline HDL-C (mg/dL) & $64.1 \pm 14.8$ & $47.8 \pm 11.0$ & $<0.001$ \\
\hline $\mathrm{HbAlc}(\%)$ & $5.5 \pm 0.7$ & $6.1 \pm 1.0$ & $<0.001$ \\
\hline Current use of antidiabetic medications & $7(7.1)$ & $6(22.2)$ & 0.02 \\
\hline \multicolumn{4}{|l|}{ Smoking status } \\
\hline Non-smoker & $60(61.2)$ & $16(59.3)$ & 0.85 \\
\hline Previous/current smoker & $38(38.8)$ & II (40.7) & \\
\hline Toothbrushing $\geq 2$ times/day & $75(76.5)$ & $20(74.1)$ & 0.79 \\
\hline Visit dentist regularly & $61(62.2)$ & $18(66.7)$ & 0.67 \\
\hline
\end{tabular}

Bold text highlights statistically significant findings.

* $P$ value for the comparison of selected characteristics by the status of MetS.

$\dagger$ All participants were aged 75 years.

be the origin of oxidative stress in patients with periodontal disease. Moreover, it was recently suggested that autophagy is an important mechanism related to inflammation. Autophagy machinery plays a crucial role in cellular homeostasis. It acts to protect cells against potential damage in association with the inflammatory process. ${ }^{37}$ It is postulated that selective autophagy of mitochondria, known as mitophagy, contributes to the maintenance of mitochondrial quality by eliminating dysfunctional or damaged mitochondria. ${ }^{38}$ Oxidative stress has been shown to induce autophagy, ${ }^{38}$ and a recent study demonstrated an enhancement of autophagy mediated by mitochondrial ROS in peripheral blood mononuclear cells from patients with periodontal disease. $^{39}$ This also supports the hypothesis that mitochondrial function is essential in inflammation-related disease, including periodontal disease. Above all, oxidative stress/mitochondrial dysfunction/autophagy mechanisms are related events in patients with chronic inflammatory diseases and are considered to be a potential link between MetS and periodontal disease.
As mentioned above, a pathogenic characteristic of MetS and periodontal disease is inflammation. Because nutrition is strongly linked to the level of inflammation, ${ }^{40}$ diet may be a common and modifiable risk factor for both MetS and periodontal disease. Dietary modification such as caloric restriction and supplementation with anti-oxidant nutrients may have a beneficial role in $\mathrm{MetS}^{41}$ and periodontal disease. ${ }^{32,40}$ More studies are needed to evaluate the effectiveness of dietary modification regarding both the quality of ingested biomolecules and the quantity of calories in controlling periodontal disease.

The current study demonstrates that participants with MetS had a significantly increased risk of periodontal disease during follow-up. Earlier research showed that periodontal disease contributed to MetS development. Morita et al. ${ }^{42}$ conducted a 4 -year longitudinal study and reported that the presence of periodontal pockets was associated with a positive conversion of $\geq 1$ MetS components (odds ratio 1.6; 95\% CI 1.1 to 2.2 ). Kobayashi et al. ${ }^{43}$ conducted a 
Table 2.

\section{Relationships of MetS and Its Components With Development of Periodontal Disease}

\begin{tabular}{|c|c|c|}
\hline \multirow[b]{2}{*}{ Component } & \multicolumn{2}{|c|}{ Outcome (development of periodontal disease) } \\
\hline & $\mathrm{RR}_{\text {crude }}(95 \% \mathrm{Cl})$ & $\mathrm{RR}_{\text {adjusted }}(95 \% \mathrm{Cl}) *$ \\
\hline No & Reference group & Reference group \\
\hline Yes & $2.23(1.03$ to 4.84$)$ & $2.58(1.17$ to 5.67$)$ \\
\hline 2 & $1.49(0.53$ to 4.18$)$ & $1.09(0.38$ to 3.11$)$ \\
\hline$\geq 3$ & $2.56(1.06$ to 6.14$)$ & $2.67(1.10$ to 6.48$)$ \\
\hline$P$ for trend & 0.04 & 0.04 \\
\hline \multicolumn{3}{|c|}{ MetS components } \\
\hline \multicolumn{3}{|c|}{ Abdominal obesity } \\
\hline Yes & $1.36(0.62$ to 2.98$)$ & $1.65(0.69$ to 3.94$)$ \\
\hline \multicolumn{3}{|c|}{ Hypertriglyceridemia } \\
\hline No & Reference group & Reference group \\
\hline Yes & 1.03 (0.43 to 2.45$)$ & $1.20(0.52$ to 2.77$)$ \\
\hline \multicolumn{3}{|l|}{ Low HDL-C } \\
\hline No & Reference group & Reference group \\
\hline Yes & $1.87(0.82$ to 4.30$)$ & 2.11 (0.96 to 4.64$)$ \\
\hline \multicolumn{3}{|c|}{ Hyperglycemia and/or medication use } \\
\hline No & Reference group & Reference group \\
\hline Yes & 1.08 (0.50 to 2.37$)$ & $0.86(0.36$ to 2.06$)$ \\
\hline
\end{tabular}

Bold text highlights statistically significant findings.

* Adjusted for sex, income, education, smoking status, number of teeth at baseline, mean CAL at baseline, pattern of visits to a dentist, and brushing frequency.

3-year longitudinal study and reported that individuals who brushed their teeth more frequently were less likely to develop MetS. The odds ratios (95\% CI) of developing MetS in individuals who brushed 2 times/day and $\geq 3$ times/day as compared with those who brushed $\leq 1$ time/day were 0.80 ( 0.49 to 1.31 ) and 0.43 (0.19 to 0.97 ), respectively. From these findings, a bidirectional relationship between MetS and periodontal disease is plausible. Additional longitudinal epidemiologic studies are needed to further evaluate the relationship between these two diseases.

The authors recognize several limitations of the study. First, there is the potential risk that the final sample analyzed may not be representative of the originally sampled cohort. The present investigation was a subset study of the Niigata Study. ${ }^{16}$ Only 454 (75.7\%) of 600 individuals originally invited to enroll in the study in 1998 underwent examinations in 2003. Over 5 years (1998 to 2003), 146 (24.3\%) of 600 dropped out of the study. Selected characteristics in 1998 were compared between the two groups, and a significantly higher percentage of in- dividuals with low education levels and individuals with a higher mean value of systolic BP were observed among those lost to follow-up. Although there were no significant differences in baseline (2003) characteristics between study participants and the individuals dropping out during the study period (2003 to 2006), it is possible that the present sample is healthier than the general population. However, the healthy cohort effect would probably bias the results toward the null hypothesis and not lead to overestimation of the observed longitudinal associations. Second, the authors analyzed only individuals with $\geq 10$ teeth; thus, the relationship between MetS and periodontitis among individuals with few teeth, including edentulous individuals, was unclear. In previous studies investigating the association of periodontal disease with MetS or diabetes, ${ }^{12,16}$ researchers excluded the individuals with $<10$ teeth from the study because of the inherent difficulties in properly assessing periodontal health in these patients. The present authors followed their decisions. Third, there may have been misclassification of study 
participants and/or incomplete control for confounding or effect modification. Information on participants' oral health status (e.g. dental plaque scores, subgingival biofilm, history of periodontal treatment), treatments for the specific lipid disorder, dietary habits, ${ }^{44}$ and exercise habits ${ }^{45}$ was not collected in this study; therefore, a number of other potentially important risk factors could not be included in the analyses. Although the most important confounders were assessed, over- or underestimation of the observed association caused by misclassification and/or residual confounding remains a risk. Finally, the study participants were restricted to the age of 75 at baseline, which could limit the generalizability of the current findings. However, increasing age is an important risk factor for both MetS and periodontal disease. ${ }^{46}$ Since the present study population consisted of older Japanese adults, the results convey the important message that MetS is associated with oral health even among older individuals and thus highlight that preventing MetS is important across the entire lifespan.

\section{CONCLUSIONS}

The findings of the present study suggest that MetS may be a risk factor for periodontal disease in community-dwelling older Japanese. Future work with larger, more diverse populations and more complete information would be necessary to substantiate the findings.

\section{ACKNOWLEDGMENTS}

This work was supported by Grants-in-Aid from the Ministry of Health and Welfare of Japan (H10-Iryo001, H13-Iryo-001, and H16-Iryo-020) and Grantsin-Aid for Young Scientists (B) (23792504 and 26861827). The authors report no conflicts of interest related to this study.

\section{REFERENCES}

1. Expert Panel on Detection, Evaluation, and Treatment of High Blood Cholesterol in Adults. Executive Summary of the Third Report of the National Cholesterol Education Program (NCEP) Expert Panel on Detection, Evaluation, and Treatment of High Blood Cholesterol in Adults (Adult Treatment Panel III). JAMA 2001;285: 2486-2497.

2. Grundy SM. Metabolic syndrome scientific statement by the American Heart Association and the National Heart, Lung, and Blood Institute. Arterioscler Thromb Vasc Biol 2005;25:2243-2244.

3. The Ministry of Health, Labour, and Welfare. National Health and Nutrition Survey 2007. Available at: http:// www0nihgojp/eiken/english/research/pdf/nhns2007pdf 2007. Accessed May 26, 2013.

4. The Ministry of Health, Labour, and Welfare. Survey of Dental Diseases, 2011. Available at: http://www.mhlw. go.jp/toukei/list/62-23. html. Accessed November 10, 2014.
5. Bullon P, Newman HN, Battino M. Obesity, diabetes mellitus, atherosclerosis and chronic periodontitis: A shared pathology via oxidative stress and mitochondrial dysfunction? Periodontol 2000 2014;64:139-153.

6. Reynolds MA. Modifiable risk factors in periodontitis: At the intersection of aging and disease. Periodontol 2000 2014;64:7-19.

7. Morita T, Ogawa Y, Takada K, et al. Association between periodontal disease and metabolic syndrome. J Public Health Dent 2009;69:248-253.

8. Andriankaja OM, Sreenivasa S, Dunford R, DeNardin E. Association between metabolic syndrome and periodontal disease. Aust Dent J 2010;55:252-259.

9. Fukui N, Shimazaki Y, Shinagawa T, Yamashita Y. Periodontal status and metabolic syndrome in middleaged Japanese. J Periodontol 2012;83:1363-1371.

10. Khader Y, Khassawneh B, Obeidat B, et al. Periodontal status of patients with metabolic syndrome compared to those without metabolic syndrome. J Periodontol 2008;79:2048-2053.

11. Kushiyama M, Shimazaki Y, Yamashita Y. Relationship between metabolic syndrome and periodontal disease in Japanese adults. J Periodontol 2009;80:1610-1615.

12. Kwon YE, Ha JE, Paik DI, Jin BH, Bae KH. The relationship between periodontitis and metabolic syndrome among a Korean nationally representative sample of adults. J Clin Periodontol 2011;38:781-786.

13. Li $P$, He L, Sha $Y Q$, Luan $Q X$. Relationship of metabolic syndrome to chronic periodontitis. J Periodontol 2009; 80:541-549.

14. Linden GJ, Lyons A, Scannapieco FA. Periodontal systemic associations: Review of the evidence. J Clin Periodontol 2013;40(Suppl. 14):S8-S19.

15. Shimazaki Y, Saito T, Yonemoto K, Kiyohara Y, lida M, Yamashita Y. Relationship of metabolic syndrome to periodontal disease in Japanese women: The Hisayama study. J Dent Res 2007;86:271-275.

16. Hirotomi T, Yoshihara A, Yano M, Ando Y, Miyazaki H. Longitudinal study on periodontal conditions in healthy elderly people in Japan. Community Dent Oral Epidemiol 2002;30:409-417.

17. Saito T, Shimazaki Y, Kiyohara Y, et al. The severity of periodontal disease is associated with the development of glucose intolerance in non-diabetics: The Hisayama study. J Dent Res 2004;83:485-490.

18. Tonetti MS, Claffey $\mathrm{N}$; European Workshop in Periodontology group $\mathrm{C}$. Advances in the progression of periodontitis and proposal of definitions of a periodontitis case and disease progression for use in risk factor research. Group C consensus report of the 5th European Workshop in Periodontology. J Clin Periodontol 2005;32(Suppl. 6):210-213.

19. Moss KL, Mauriello S, Ruvo AT, Offenbacher S, White RP Jr., Beck JD. Reliability of third molar probing measures and the systemic impact of third molar periodontal pathology. J Oral Maxillofac Surg 2006;64: 652-658.

20. Ninomiya T, Kubo M, Doi Y, et al. Impact of metabolic syndrome on the development of cardiovascular disease in a general Japanese population: The Hisayama study. Stroke 2007;38:2063-2069.

21. Veeranna V, Ramesh K, Zalawadiya SK, et al. Glycosylated hemoglobin and prevalent metabolic syndrome in nondiabetic multiethnic U.S. adults. Metab Syndr Relat Disord 2011;9:361-367.

22. Bullon P, Morillo JM, Ramirez-Tortosa MC, Quiles JL, Newman HN, Battino M. Metabolic syndrome and 
periodontitis: Is oxidative stress a common link? J Dent Res 2009;88:503-518.

23. Hotamisligil GS. Inflammation and metabolic disorders. Nature 2006;444:860-867.

24. Ishikawa S, Kayaba K, Gotoh T, Nakamura Y, Kajii E. Metabolic syndrome and C-reactive protein in the general population: JMS Cohort Study. Circ J 2007; 71:26-31.

25. Salmenniemi U, Ruotsalainen E, Pihlajamäki J, et al. Multiple abnormalities in glucose and energy metabolism and coordinated changes in levels of adiponectin, cytokines, and adhesion molecules in subjects with metabolic syndrome. Circulation 2004;110:38423848

26. Ramirez-Tortosa MC, Quiles JL, Battino M, et al. Periodontitis is associated with altered plasma fatty acids and cardiovascular risk markers. Nutr Metab Cardiovasc Dis 2010;20:133-139.

27. Nishimura F, Iwamoto Y, Mineshiba J, Shimizu A, Soga Y, Murayama Y. Periodontal disease and diabetes mellitus: The role of tumor necrosis factor-alpha in a 2-way relationship. J Periodontol 2003;74:97-102.

28. Saito T, Shimazaki Y, Sakamoto M. Obesity and periodontitis. N Engl J Med 1998;339:482-483.

29. Saito T, Yamaguchi N, Shimazaki Y, et al. Serum levels of resistin and adiponectin in women with periodontitis: The Hisayama study. J Dent Res 2008;87:319-322.

30. Kowaltowski AJ, de Souza-Pinto NC, Castilho RF, Vercesi AE. Mitochondria and reactive oxygen species. Free Radic Biol Med 2009;47:333-343.

31. Ilkun O, Boudina S. Cardiac dysfunction and oxidative stress in the metabolic syndrome: An update on antioxidant therapies. Curr Pharm Des 2013;19:48064817.

32. Chapple IL, Matthews JB. The role of reactive oxygen and antioxidant species in periodontal tissue destruction. Periodontol 2000 2007;43:160-232.

33. Ohnishi T, Bandow K, Kakimoto K, Machigashira M, Matsuyama T, Matsuguchi T. Oxidative stress causes alveolar bone loss in metabolic syndrome model mice with type 2 diabetes. J Periodontal Res 2009;44:43-51.

34. D'Aiuto F, Nibali L, Parkar M, Patel K, Suvan J, Donos N. Oxidative stress, systemic inflammation, and severe periodontitis. J Dent Res 2010;89:1241-1246.

35. Canakçi CF, Canakçi V, Tatar A, et al. Increased salivary level of 8-hydroxydeoxyguanosine is a marker of premature oxidative mitochondrial DNA damage in gingival tissue of patients with periodontitis. Arch Immunol Ther Exp (Warsz) 2009;57:205-211.
36. Bullon P, Cordero MD, Quiles JL, Morillo JM, del Carmen Ramirez-Tortosa M, Battino M. Mitochondrial dysfunction promoted by Porphyromonas gingivalis lipopolysaccharide as a possible link between cardiovascular disease and periodontitis. Free Radic Biol Med 2011;50:1336-1343.

37. Bugger H, Abel ED. Mitochondria in the diabetic heart. Cardiovasc Res 2010;88:229-240.

38. Hariharan N, Zhai P, Sadoshima J. Oxidative stress stimulates autophagic flux during ischemia/reperfusion. Antioxid Redox Signal 2011;14:2179-2190.

39. Bullon P, Cordero MD, Quiles JL, et al. Autophagy in periodontitis patients and gingival fibroblasts: Unraveling the link between chronic diseases and inflammation. BMC Med 2012;10:122.

40. Dawson DR 3rd, Branch-Mays G, Gonzalez OA, Ebersole JL. Dietary modulation of the inflammatory cascade. Periodontol 2000 2014;64:161-197.

41. Ford ES, Mokdad AH, Giles WH, Brown DW. The metabolic syndrome and antioxidant concentrations: Findings from the Third National Health and Nutrition Examination Survey. Diabetes 2003;52:2346-2352.

42. Morita T, Yamazaki Y, Mita A, et al. A cohort study on the association between periodontal disease and the development of metabolic syndrome. J Periodontol 2010;81:512-519.

43. Kobayashi Y, Niu K, Guan L, et al. Oral health behavior and metabolic syndrome and its components in adults. J Dent Res 2012;91:479-484.

44. Hujoel P. Dietary carbohydrates and dental-systemic diseases. J Dent Res 2009;88:490-502.

45. Sanders AE, Slade GD, Fitzsimmons TR, Bartold PM. Physical activity, inflammatory biomarkers in gingival crevicular fluid and periodontitis. J Clin Periodontol 2009;36:388-395.

46. Urashima $M$. Prevalence of metabolic syndrome in 22,892 Japanese population and its association with life style. Japan Med Assoc J 2005;48:441-450.

Correspondence: Dr. Masanori Iwasaki, Division of Community Oral Health Development, Kyushu Dental University, 2-6-1 Manazuru, Kokurakita-ku, Kitakyushu 803-8580 Japan. Fax: +81-93-591-7736; e-mail: r14iwasaki@fa. kyu-dent.ac.jp.

Submitted July 3, 2014; accepted for publication November 14, 2014. 